\title{
Avaliação do ciclo de vida na fabricação de clínquer em diferentes cenários energéticos
}

A técnica do coprocessamento tem sido empregada mundialmente na busca da redução do consumo de combustíveis fósseis e matérias-primas tradicionais na fabricação de clínquer, utilizando-se de resíduos industriais para este fim. No entanto, é preciso avaliar o desempenho ambiental desta prática. O objetivo do trabalho foi comparar os impactos ambientais de três cenários distintos quanto ao percentual de combustíveis tradicionais e alternativos no mix energético para a produção de clínquer. A metodologia utilizada foi a avaliação do ciclo de vida. A unidade funcional adotada foi a produção de uma tonelada de clínquer. O método de Avaliação de Impacto escolhido foi o ReCiPe midpoint. Os resultados da caracterização indicaram que as categorias Toxicidade Humana, Formação de Oxidantes Fotoquímicos, Formação de Material Particulado e Acidificação Terrestre sofreram redução do impacto com o uso dos blends de resíduos; por outro lado, a maioria das categorias obteve um aumento do impacto conforme aumento do percentual de substituição. A normalização apontou a Toxicidade Humana como o principal impacto da atividade e a sua maior redução em $62 \%$ no cenário B. Conclui-se que devido à periculosidade dos resíduos empregados no caso estudado o coprocessamento no Brasil deve incorporar medidas de mitigação, dentre elas, o aumento da participação de resíduos de biomassa nos blends para que possa avançar em projeções mais ambiciosas de substituição térmica dos combustíveis tradicionais.

\section{Life cycle assessment in the manufacture of clinker in different energy scenarios}

\begin{abstract}
The co-processing technique has been used worldwide in order to reduce the consumption of fossil fuels and traditional raw materials in the manufacture of clinker, using industrial waste for this purpose. However, it is necessary to evaluate the environmental performance of this practice. The objective of the work was to compare the environmental impacts of three different scenarios regarding the percentage of traditional and alternative fuels in the energy mix for the production of clinker. The methodology used was the life cycle assessment. The functional unit adopted was the production of one ton of clinker. The Impact Assessment method chosen was the ReCiPe midpoint. The results of the characterization indicated that the categories Human Toxicity, Formation of Photochemical Oxidizers, Formation of Particulate Material and Terrestrial Acidification suffered a reduced impact with the use of waste blends; on the other hand, most categories had an increase in impact as the percentage of substitution increased. Normalization pointed to Human Toxicity as the main impact of the activity and its greatest reduction by $62 \%$ in B scenario. It is concluded that due to the hazardous nature of the waste used in the case studied, co-processing in Brazil must incorporate mitigation measures, among them, the increase in the share of biomass residues in the blends so that it can move forward with more ambitious projections for the thermal substitution of traditional fuels.
\end{abstract}

Keywords: Life Cycle Assessment; Environmental impacts; Clinker; Waste; Human Toxicity.

Topic: Engenharia Ambiental

Reviewed anonymously in the process of blind peer.
Received: 03/06/2021

Approved: 27/06/2021

David Barreto de Aguiar (iD)

Universidade do Estado do Rio de Janeiro, Brasil

http://lattes.cnpq.br/7229051875249959

http://orcid.org/0000-0003-1233-0253

david.aguiar@ifrj.edu.br

Ubirajara Aluizio de Oliveira Mattos (DD)

Universidade do Estado do Rio de Janeiro, Brasil

http://lattes.cnpq.br/4965089463046103

http://orcid.org/0000-0002-2689-2110

ubirajaraaluizio@yahoo.com.br

Victor Paulo Peçanha Esteves

Universidade Federal do Rio de Janeiro, Brasil

http://lattes.cnpq.br/7858887034547590

http://orcid.org/0000-0002-6812-4558

victoresteves@poli.ufri.br

\section{Referencing this:}

AGUIAR, D. B.; MATTOS, U. A. O.; ESTEVES, V. P. P.. Avaliação do ciclo de vida na fabricação de clínquer em diferentes cenários energéticos. Revista Ibero Americana de Ciências Ambientais, v.12, n.6, p.387394, 2021. DOI: http://doi.org/10.6008/CBPC21796858.2021.006.0032 


\section{INTRODUÇÃO}

Estima-se que o setor de produção de cimento em todo o mundo tenha sido responsável pela emissão atmosférica de 403 milhões de toneladas de carbono (MtC) em 2017, sendo que, desse total, 68 MtC tenham sido originadas da queima de combustíveis fósseis como carvão, óleo e gás. Essa indústria é responsável por quatro por cento (4\%) das emissões mundiais de CO2 (LE QUÉRÉ et al., 2018).

Segundo Rodrigues et al. (2017), do ponto de vista de um processo efetivo de desenvolvimento, os projetos intensivos em energia destinam-se ao aproveito simultâneo de recursos minerais e energéticos.

A técnica do coprocessamento tem sido empregada mundialmente com a finalidade de reduzir o consumo de combustível fóssil e matérias-primas na fabricação de cimento. Trata-se da utilização de rejeitos de atividades industriais, pneus e até mesmo resíduos sólidos urbanos como combustíveis alternativos e matérias-primas nesse processo produtivo (LAMAS et al., 2013).

De acordo com Tristão et al. (2017), a queima de resíduos perigosos como o óleo lubrificante usado só pode acontecer de forma controlada após remoção de compostos clorados e metais pesados.

A comunidade europeia tem realizado várias projeções para a substituição de combustíveis fósseis para a produção de cimento, assumindo a proporção de 50-50\% entre combustíveis fósseis e alternativos para 27 países da União Europeia UE27, em 2030 (MOYA et al., 2011), porém, os riscos no preparo dos resíduos para o coprocessamento são minimizados através da automação dos processos (MILANEZ et al., 2009).

Stafford et al. (2016) realizaram estudo numa planta de produção de cimento brasileira que utilizou um percentual de nove por cento (9\%) na substituição de combustível tradicional por alternativo, porém encontrou altos valores para toxicidade humana utilizando a Avaliação de Ciclo de Vida. A categoria Toxicidade Humana não está inclusa na Declaração de Produtos (EPD) do cimento na Europa por considerar que não há uma modelo confiável para metais.

Milanez et al. (2009) discutiram a coincineração de resíduos em fábricas de cimento no Brasil, bem como seus impactos sobre a saúde humana e o meio ambiente. Foi constatado, entre os trabalhadores dessas indústrias, danos à saúde humana, como as dermatites causadas pela contaminação do cimento por cromo IV e VI, além dos riscos sanitários como a exposição a vapores e odores das diversas etapas de mistura e homogeneização de resíduos perigosos, conhecidas como blendagem.

Como bem recorda Milan (2015), muitos líquidos, óleos e produtos químicos como tintas e solventes, além de óleos refrigerantes e de motor, são tóxicos.

Pinto Júnior et al. (2009) analisaram as condições de trabalho a partir dos relatos dos trabalhadores que adoeceram nas atividades de coprocessamento de resíduos tóxicos em fornos de cimenteiras brasileiras e identificaram falhas na observação do risco e no monitoramento dos impactos ambientais e à saúde humana.

Rocha et al. (2011) mencionam que o coprocessamento é um campo amplo de investigação para a (ACV) e indica que cada tipo de resíduo coprocessado deve ser objeto de estudo, uma vez que suas 
características físicas e químicas podem alterar os resultados; porém, tal aspecto, apresenta uma oportunidade de aprofundamento do conhecimento dos processos e impactos da indústria do prétratamento de resíduos para coprocessamento.

Lemos (2009) concluiu, após um determinado estudo de caso, que não houve alteração química do clínquer com o uso de até $30 \%$ de blend de resíduos na atividade. No entanto, poucos ainda foram os estudos científicos desenvolvidos sobre os impactos ambientais gerados à saúde humana através do coprocessameto na indústria do cimento (LAMAS et al., 2013).

$\mathrm{Na}$ literatura científica sobre o assunto não foi encontrada nenhuma referência nacional que considerasse um aprofundamento no conhecimento dos impactos pela avaliação do ciclo de vida quanto à situação atual e à ampliação do uso de blends de resíduos perigosos, tendo em vista a diversidade de resíduos industriais utilizados na sua composição.

Em face dos argumentos expostos, o presente trabalho justifica-se pela necessidade de investigar os impactos ocasionados por intermédio da possível ampliação do percentual de participação dos resíduos industriais e outros passivos ambientais para coprocessamento na produção de clínquer, na indústria de cimento.

\section{METODOLOGIA}

\section{Objetivo e Escopo}

Os dados do inventário foram obtidos, em primeiro plano, baseado em perfil de três plantas situadas no polo cimenteiro do Município de Cantagalo, Região Serrana do Estado do Rio de Janeiro, com média anual de produção de 530.330 t de clínquer, baseada entre os anos de 2010 e 2016, por meio de duas fontes: 1å) através da consulta ao Portal Nacional do Licenciamento (PNLA), os meses de novembro e dezembro de 2020, utilizando a expressão: "coprocessamento de resíduos", no campo destinado ao tipo de atividade econômica (PNLA, 2021); 2a) no Portal do Instituto Estadual do Ambiente do Rio de Janeiro (INEA), através do número do processo de licenciamento ambiental, obtidos no PNLA, foram consultados diversos documentos, como: registros de balanço de massa dos fornos de cimento, vistorias, pareceres, relatórios de áreas contaminadas e auditorias ambientais que continham informações sobre emissões líquidas e gasosas do processo, bem como os diversos tipos de resíduos industriais utilizados na preparação dos compostos energéticos conhecidos como blends. Cabe registrar que a busca de informação contemplou documentos que se enquadram na categoria "Literatura Cinzenta" (BOTELHO et al., 2015):

Diz respeito a publicações não convencionais e não comerciais, semipublicadas, difíceis de encontrar em canais tradicionais de distribuição [...]. Apresentam informação e conhecimento altamente atualizados e mais detalhados, alcançam um público reduzido e não são determinadas apenas por interesses comerciais.

Os blends são produzidos em unidades de tratamento, mistura e homogeneização, conhecidas no Brasil como blendeiras. Os percentuais distintos dos resíduos utilizados na produção dos blends sólidos, caracterizados de 10, 50 ou até $100 \mathrm{~mm}$ granulometria, são os seguintes: solo contaminado (39,83\%), borra oleosa $(16,35 \%)$, graxas $(8,89 \%)$, resíduos químicos e reagentes diversos $(6,59 \%)$, areia contaminada $(4,08 \%)$, 
borra de tintas - base de água (2,96\%), base solvente $(2,29 \%)$-, lodo de ETE (2\%), resinas $(1,94 \%)$, brita contaminada $(1,87 \%)$, demais resíduos com menos de um por cento (1\%) na composição $(11,59 \%)$ (INEA, 2019).

Já os resíduos industriais que formam os blends líquidos apresentaram a seguinte distribuição percentual: lubrificantes $(39,43 \%)$, solventes $(23,54 \%)$, etanol $(9,98 \%)$, sulfonato de alquibenzeno $(5,33 \%)$, álcoois etoxilados $(5,25 \%)$, sulfato de ferro $(4,25 \%)$, diesel $(2,69 \%)$, ácido fosfórico $(2,04 \%)$, formaldeído $(1,61 \%)$, ácido graxo $(1,32 \%)$, óleos $(1,32 \%)$, água oleosa $(1,01 \%)$ e os demais resíduos líquidos menores que 1\% na composição $(5,23 \%)$. Foi computada, também, a entrada de agentes estruturantes e químicos, como maravalha (serragem) e cal virgem, respectivamente, que são misturados aos resíduos na blendagem (INEA, 2019).

Foi utilizada a metodologia da ACV conforme as normas técnicas NBR ISO 14040 e 14044 (ABNT, 2009ab), com o objetivo de comparar os impactos ambientais de três cenários distintos quanto ao percentual de combustíveis tradicionais e alternativos no mix energético para a produção de clínquer.

As fronteiras de sistema não computaram os impactos do transporte e do pré-tratamento de resíduos, realizada em unidade industrial específica (blendeira), levando em consideração apenas a queima dos resíduos transformados em blends como combustível alternativo. Dados de entrada como matériasprimas para produção de clínquer e o consumo de eletricidade foram obtidos com base no perfil das plantas estudadas e repetidos nos três cenários. As emissões atmosféricas, líquidas e a geração de resíduos foram consideradas. A abordagem realizada foi a do portão-ao-portão. A unidade funcional adotada foi a produção de uma tonelada de clínquer.

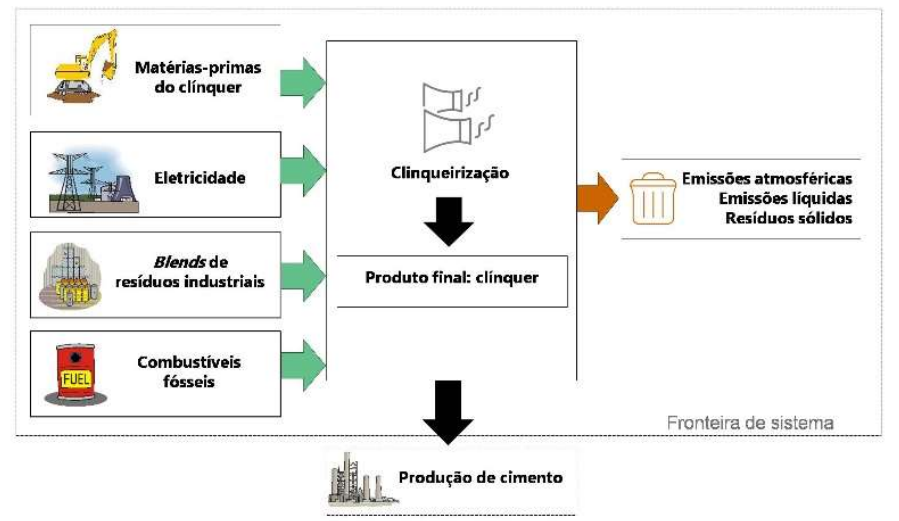

Figura 1: Delimitação da Fronteira de Sistema.

O cenário A considerou a clinqueirização com a utilização de $100 \%$ coque de petróleo, como combustível dos fornos; o cenário B: $91 \%$ de coque de petróleo e $9 \%$ de combustíveis alternativos (blends), representando um cenário atual do percentual de substituição neste polo cimenteiro; por fim, o cenário C: $70 \%$ coque e $30 \%$ de blends, considerando uma possível projeção futura no país (LEMOS, 2009). Nos cenários B e C os blends sólidos correspondem a $90 \%$ dos resíduos alternativos, obedecendo ao que foi encontrado nas fontes de consulta.

A caracterização dos impactos na etapa de Avaliação do Impacto do Ciclo de Vida (AICV) foi realizada 
utilizando os fatores contidos no método ReCiPe midpoint (H) v. 1.04 (HUIJBREGTS et al., 2017), calculado com auxílio do software SimaPro v.7.2 com data server, considerando as categorias de impacto relevantes para o estudo: Mudanças Climáticas (MC), Depleção do Ozônio (DO), Toxicidade Humana (TH), Formação de Oxidantes Fotoquímicos (FOF), Formação de Material Particulado (FMP), Radiação Ionizante (RI), Acidificação Terrestre (AT), Eutrofização da Água Doce (EA), Ecotoxicidade Terrestre (ET) e da Água (ETA), Transformação da Terra Natural (TTN), Depleção da Água (DA), Mineral (DM) e Fóssil (DF). A seguir foi realizado o procedimento de normalização, utilizado o conjunto de Normalização World ReCiPe H.

\section{RESULTADOS E DISCUSSÃO}

\section{Inventário do Ciclo de Vida}

A Figura 1 apresenta o ICV com principais fluxos de entrada e saída dos cenários de coprocessamento para a produção de 1 t de clínquer. Como foi utilizada também a base de dados do SimaPro Data Server, resultando em muitas entradas e saídas no inventário, foi feito um corte mássico que desconsiderou as substâncias que contribuíram com menos de $5 \%$ para o impacto.

Tabela 1: Fluxos para produção de $1 \mathrm{t}$ de clínquer nos diferentes cenários.

\begin{tabular}{|c|c|c|c|c|c|c|}
\hline \multirow[t]{2}{*}{ Fluxo } & \multirow[t]{2}{*}{ Compartimento } & \multirow[b]{2}{*}{ SUBSTÂNCIAS } & \multirow[b]{2}{*}{ Unid. } & \multicolumn{3}{|l|}{ Cenários } \\
\hline & & & & A & B & C \\
\hline \multirow{13}{*}{ Entradas } & \multirow{13}{*}{ Matéria-prima } & Transformação da floresta & $\mathrm{cm}^{2}$ & $2,58 \mathrm{E}+01$ & $2,18 \mathrm{E}+03$ & $7,20 \mathrm{E}+03$ \\
\hline & & Água & $\mathrm{m}^{3}$ & $1,46 \mathrm{E}-01$ & $2,61 \mathrm{E}+00$ & $8,39 E+00$ \\
\hline & & Alumínio & $\mathrm{kg}$ & $4,07 E+01$ & $4,07 E+01$ & $4,07 E+01$ \\
\hline & & Cromo & $\mathrm{g}$ & $2,70 \mathrm{E}+00$ & $1,48 \mathrm{E}+01$ & $4,31 E+01$ \\
\hline & & Ferro & $\mathrm{Oz}$ & $1,93 E+00$ & $2,32 E+01$ & $7,27 E+01$ \\
\hline & & Manganês & g & $6,33 \mathrm{E}-01$ & $1,00 E+01$ & $3,20 E+01$ \\
\hline & & Níquel & $\mathrm{g}$ & $6,55 E+00$ & $3,76 E+01$ & $1,10 E+02$ \\
\hline & & Estanho & g & $1,86 \mathrm{E}-03$ & $4,24 \mathrm{E}-01$ & $1,41 E+00$ \\
\hline & & Carvão mineral & kg & $2,51 \mathrm{E}+02$ & $2,28 \mathrm{E}+02$ & $1,76 \mathrm{E}+02$ \\
\hline & & Gás natural & $m^{3}$ & $1,10 \mathrm{E}+00$ & $7,27 \mathrm{E}+00$ & $2,17 E+01$ \\
\hline & & Óleo cru & kg & $2,37 E+00$ & $9,62 E+00$ & $2,65 E+01$ \\
\hline & & Dióxido de Carbono, fóssil & $\mathrm{kg}$ & $4,44 \mathrm{E}+01$ & $7,68 \mathrm{E}+01$ & $1,52 E+02$ \\
\hline & & Metano & $\mathrm{Oz}$ & $3,10 E+01$ & $4,78 E+01$ & $8,70 E+01$ \\
\hline \multirow{19}{*}{ Saídas } & \multirow{13}{*}{$\mathrm{Ar}$} & Bromotrifluorometano & $\mu \mathrm{g}$ & $2,57 E+00$ & $8,99 \mathrm{E}+01$ & $2,94 \mathrm{E}+02$ \\
\hline & & Bromoclorodifluorometano & $\mu \mathrm{g}$ & $1,86 \mathrm{E}+00$ & $1,44 \mathrm{E}+02$ & $4,77 E+02$ \\
\hline & & Bromometano & $\mu g$ & $7,48 \mathrm{E}+00$ & $7,59 \mathrm{E}+00$ & $7,85 \mathrm{E}+00$ \\
\hline & & Clorodifluorometano & $\mathrm{g}$ & 4,33E-05 & $2,99 E+00$ & $9,98 E+00$ \\
\hline & & Diclorodifluorometano & $\mathrm{mg}$ & $1,46 \mathrm{E}-03$ & $1,18 \mathrm{E}+02$ & $3,92 \mathrm{E}+02$ \\
\hline & & Tetraclorometano & $\mathrm{mg}$ & $1,69 \mathrm{E}-03$ & $8,80 E+01$ & $2,93 E+02$ \\
\hline & & Mercúrio & $\mathrm{mg}$ & $4,93 E+01$ & $3,49 E+00$ & $8,10 E+00$ \\
\hline & & Óxidos de Nitrogênio & $\mathrm{oz}$ & $2,65 E+02$ & $1,15 E+01$ & $2,18 \mathrm{E}+01$ \\
\hline & & COVNM & $\mathrm{g}$ & $6,16 \mathrm{E}+02$ & $5,96 \mathrm{E}+02$ & $5,51 E+02$ \\
\hline & & Dióxido de Enxofre & $\mathrm{Oz}$ & $1,58 \mathrm{E}+02$ & $8,89 E+00$ & $2,20 E+01$ \\
\hline & & Óxidos de Enxofre & $\mathrm{g}$ & $7,82 \mathrm{E}+02$ & $7,21 E+02$ & $5,58 \mathrm{E}+02$ \\
\hline & & Carbono 14 & $\mathrm{~Bq}$ & $5,85 E+00$ & $1,62 E+02$ & $5,26 E+02$ \\
\hline & & Radônio 222 & $\mathrm{kBq}$ & $1,00 \mathrm{E}+02$ & $3,25 E+03$ & $1,06 E+04$ \\
\hline & \multirow{5}{*}{ Água } & Fosfato & $\mathrm{g}$ & 4,74E-01 & $3,45 E+01$ & $1,14 \mathrm{E}+02$ \\
\hline & & Fósforo & g & $1,04 \mathrm{E}-04$ & $7,16 \mathrm{E}-01$ & $2,39 E+00$ \\
\hline & & Selenium & g & $1,18 \mathrm{E}+01$ & $1,19 \mathrm{E}+01$ & $1,20 E+01$ \\
\hline & & Níquel, íon & g & 4,76E-02 & $8,64 \mathrm{E}-01$ & $2,77 \mathrm{E}+00$ \\
\hline & & Cipermetrina & $\mathrm{mg}$ & $2,50 \mathrm{E}-05$ & $8,77 E+00$ & $2,92 E+01$ \\
\hline & Solo & Fósforo & $\mathrm{mg}$ & $1,29 E+01$ & $7,97 E+01$ & $2,36 \mathrm{E}+02$ \\
\hline
\end{tabular}

\section{Avaliação de Impacto do Ciclo de Vida}

A Tabela 2 apresenta o resultado da caracterização dos impactos dos valores do ICV pelo método ReCiPe. 
Tabela 2: Caracterização dos impactos ambientais nos diferentes cenários.

\begin{tabular}{lllll} 
Categoria de Impacto & Unidade & CENÁRIOS & A & B \\
\hline MC & kg CO2 eq & $6,67 \mathrm{E}+01$ & $1,28 \mathrm{E}+02$ & $2,72 \mathrm{E}+02$ \\
DO & kg CFC-11 eq & $5,30 \mathrm{E}-08$ & $3,43 \mathrm{E}-04$ & $1,14 \mathrm{E}-03$ \\
TH & kg 1,4-DB eq & $3,48 \mathrm{E}+02$ & $1,44 \mathrm{E}+02$ & $1,70 \mathrm{E}+02$ \\
FPF & kg NMVOC & $8,58 \mathrm{E}+00$ & $1,08 \mathrm{E}+00$ & $1,48 \mathrm{E}+00$ \\
FMP & kg PM10 eq & $2,83 \mathrm{E}+00$ & $3,20 \mathrm{E}-01$ & $5,02 \mathrm{E}-01$ \\
RI & kg U235 eq & $1,74 \mathrm{E}-01$ & $5,37 \mathrm{E}+00$ & $1,75 \mathrm{E}+01$ \\
TA & kg SO2 eq & $9,53 \mathrm{E}+00$ & $1,25 \mathrm{E}+00$ & $1,75 \mathrm{E}+00$ \\
EA & kg P eq & $1,70 \mathrm{E}-04$ & $1,22 \mathrm{E}-02$ & $4,03 \mathrm{E}-02$ \\
ET & kg 1,4-DB eq & $6,15 \mathrm{E}-03$ & $1,38 \mathrm{E}-01$ & $4,55 \mathrm{E}-01$ \\
ETA & kg 1,4-DB eq & $1,06 \mathrm{E}+00$ & $1,35 \mathrm{E}+00$ & $2,03 \mathrm{E}+00$ \\
TTN & m2 & $5,86 \mathrm{E}-04$ & $4,53 \mathrm{E}-02$ & $1,50 \mathrm{E}-01$ \\
DA & m3 & $1,53 \mathrm{E}-01$ & $2,66 \mathrm{E}+00$ & $8,52 \mathrm{E}+00$ \\
DM & kg Fe eq & $3,95 \mathrm{E}+00$ & $7,73 \mathrm{E}+00$ & $1,65 \mathrm{E}+01$ \\
DF & kg oil eq & $1,62 \mathrm{E}+02$ & $1,66 \mathrm{E}+02$ & $1,75 \mathrm{E}+02$ \\
\hline
\end{tabular}

A Figura 2 apresenta o desempenho ambiental a partir da AICV que comparou os cenários. Notou-se que os impactos mitigados nos cenários B e C foram TH, FOF, FMP e AT, em relação ao cenário A. Por outro lado, verificou-se que a elevação do percentual de nove (9\%) para $30 \%$ acarretou no aumento do impacto nas demais categorias, conferindo ao cenário A o melhor desempenho na maioria delas.

A razão para os resultados não promissores da substituição térmica dos cenários B e C está na característica dos resíduos utilizados. Segundo a classificação da NBR 10.004 a maioria dos resíduos industriais utilizada no coprocessamento foi classificada como da Classe I (perigosos) que, segundo a NBR 10004, são resíduos que apresentam maiores riscos à saúde e ao meio ambiente (ABNT, 2004).

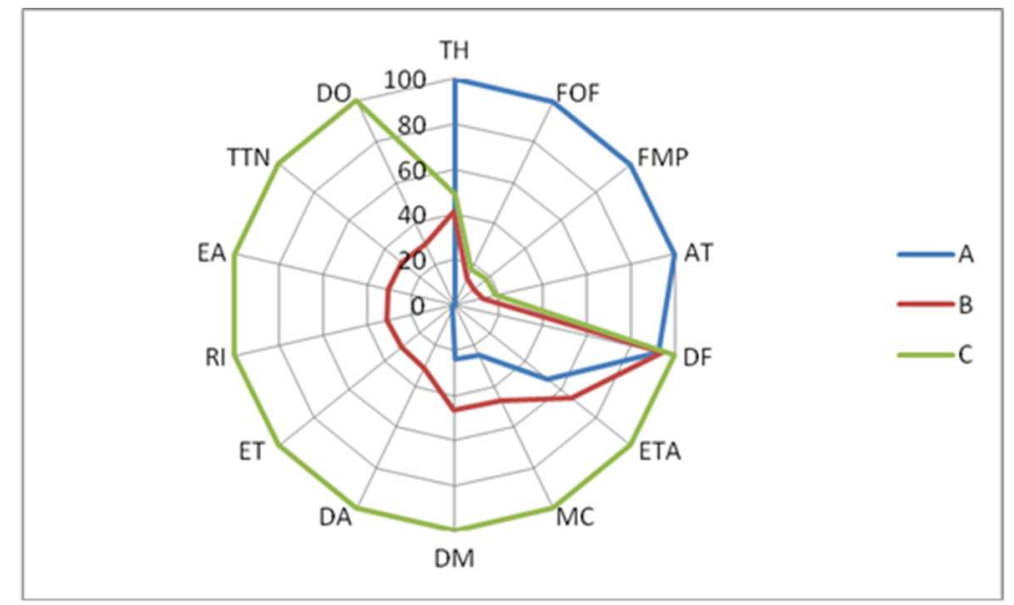

Figura 2: Comparação do Desempenho Ambiental dos cenários pela ACV.

A Normalização indicou que a TH é o principal impacto ambiental em todos os cenários, responsável $56 \%, 41 \%$ e $34 \%$ pelo impacto total em A, B e C, respectivamente, porém, apresentou uma redução de $62 \%$ e 52\%, especificamente nesta categoria, nos cenários B e C. No cenário A, a TH é ocasionada, principalmente, pela emissão de chumbo $(\mathrm{Pb})$ para o ar, correspondendo a $58 \%$ deste impacto.

Nos cenários $\mathrm{B}$ e $\mathrm{C}$ as emissões de $\mathrm{Pb}$ para o ar são praticamente eliminadas, porém, passou a predominar o Selênio (Se) emitido para a água como responsável por $87 \%$ e $75 \%$ da TH, respectivamente. No cenário $\mathrm{A}$, as emissões de $\mathrm{Pb}$ são originadas da queima dos combustíveis fósseis no coprocessamento. Já em B e C o Se foi oriundo dos efluentes emitidos pelos blends. Resíduos predominantes na composição dos blends, como as borras de tintas são prováveis fontes desse metal. 
Com relação aos impactos na saúde, o $\mathrm{Pb}$ pode ocasionar males neurológicos, hepáticos, hematológico, reprodutores, renais e até mesmo o câncer (CETESB, 2020a). Já o Se pode ocasionar efeitos adversos, como distúrbios gastrointestinais e alterações dos nervos periféricos (CETESB, 2020b). No caso específico, o risco de adoecimento é maior para os trabalhadores das cimenteiras pelo contato direto com os resíduos e, em seguida, para a população circundante às áreas do empreendimento, por meio das emissões diretamente para o ar e para a água.

Notou-se, também, uma significante redução dos impactos das categorias FOF, FMP, e AT nos cenários B e $C$ em comparação ao cenário $A$. Ao contrário disso, houve aumento da $E A, E T, M C, O D$ e DF nos cenários B e C, e da ETA e DM, apenas no cenário C. Na categoria DA o impacto foi nulo em todos os cenários. Por sua vez, nas categorias RI e TTN o impacto foi praticamente insignificante nos três cenários (Figura 3).

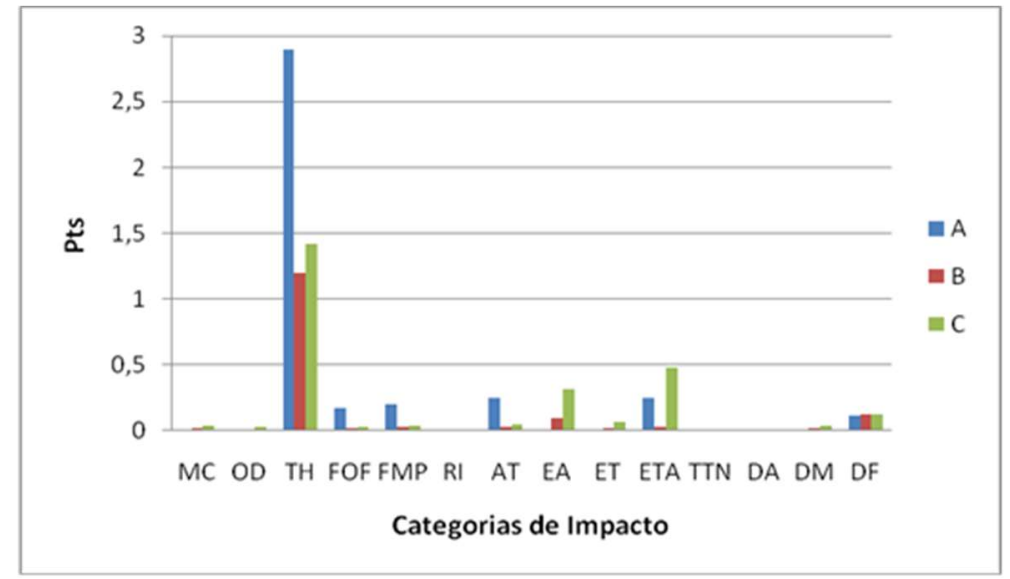

Figura 3: Comparativo dos impactos nos cenários estudados.

\section{CONCLUSÕES}

Com base no comparativo realizado, que apresentou diferentes cenários de coprocessamento, contendo a substituição parcial de combustíveis tradicionais por alternativos no mix energético, na produção de clínquer, concluiu-se que a indústria do cimento precisa ter cautela no que tange ao aumento percentual dessa substituição térmica de combustíveis fósseis por alternativos, tendo em vista a possibilidade do surgimento de impactos adversos.

O atual percentual de substituição de nove por cento já se apresenta como um limitante para os ganhos ambientais do coprocessamento, por se tratar de resíduos industriais com alto potencial poluidor e periculosidade. Uma projeção ambiciosa de $30 \%$ poderá ser planejada, com a maior participação de resíduos menos tóxicos que atendam à demanda energética requerida e fontes de biomassa que outrora aparecem com pequeno percentual na lista de resíduos coprocessados.

Outras medidas importantes são: 1a) adoção de critérios técnicos mais rigorosos de classificação dos resíduos industriais que poderão ser empregados no coprocessamento, seja na etapa da preparação dos blends, seja na queima nos fornos de clínquer; 2a) aprofundamento no conhecimento dos potenciais impactos dos processos de preparação dos blends; 3a) a automação dos processos de pré-tratamento; 4a) a revisão da legislação; e 5a) uma maior efetividade no controle e na fiscalização por parte dos órgãos 
ambientais, que poderão exigir melhoria dos processos, culminando na redução dos impactos ao meio ambiente e à saúde humana.

À guisa de recomendação, sugere-se a elaboração de novos estudos de ACV que abordem os impactos da cadeia do coprocessamento, incluindo a preparação dos resíduos na blendagem e o transporte entre as unidades de processo.

\section{REFERÊNCIAS}

ABNT. Associação Brasileira de Normas Técnicas. ABNT NBR 10004: resíduos sólidos - classificação. 2 ed. Rio de Janeiro: ABNT, 2004.

ABNT. Associação Brasileira de Normas Técnicas. ABNT NBR ISO 14040: gestão ambiental - Avaliação do ciclo de vida princípios e estrutura. Rio de Janeiro: ABNT, 2009a.

ABNT. Associação Brasileira de Normas Técnicas. ABNT NBR ISO 14044: gestão ambiental - avaliação do ciclo de vida requisitos e orientações. Rio de Janeiro: ABNT, 2009b.

BOTELHO, R. G.; OLIVEIRA, C. C.. Literaturas branca e cinzenta: uma revisão conceitual. Ciência da Informação, Brasília, v.44, n.3, p.501-513, 2015.

CETESB. Companhia Ambiental do Estado de São Paulo. Ficha de informação toxicológica: chumbo. São Paulo: Divisão de Toxicologia Humana e Saúde Ambiental, 2020a.

CETESB. Companhia Ambiental do Estado de São Paulo. Ficha de informação toxicológica: selênio. São Paulo: Divisão de Toxicologia Humana e Saúde Ambiental, 2020 b.

HUIJBREGTS, M. A. J.; STEINMANN, Z. J. N.; ELSHOUT, P. M. F.; STAM, G.; VERONES, F.; VIEIRA M.; ZIJP, M.; HOLLANDER, A.; VAN ZELM, R.. ReCiPe2016: a harmonized life cycle impact assessment method at midpoint and endpoint level. The International Journal of Life Cycle Assessment, v.22, p.138-147, 2017.

INEA. Instituto Estadual do Ambiente. Portal do Licenciamento. Rio de Janeiro: INEA, 2020.

LAMAS, W. Q.; PALAU, J. C. F.; CAMARGO, J. R.. Waste materials co-processing in cement industry: ecological efficiency of waste reuse. Renewable and Sustainable Energy Reviews, v.19, p.200-207, 2013. DOI: https://doi.org/10.1016/j.rser.2012.11.015

LEMOS, W. D.. Impactos do coprocessamento de resíduos em um forno de clínquer. Monografia (Bacharelado em Engenharia de Produção) - Centro Universitário de Formiga, Formiga, 2009.

LE QUÉRÉ, C. et al. The global carbon budget 2018. Earth System Science Data, v.10, n.4, p.2141-2194, 2018. DOI: https://doi.org/10.5194/essd-10-2141-2018
MILAN, M.. Reciclagem de materiais: empresa e conceito. São Paulo: SENAI-SP, 2017.

MILANEZ, B.; FERNANDES, L. O.; PORTO, M. F. S.. A coincineração de resíduos em fornos de cimento: riscos para a saúde e o meio ambiente. Ciência \& Saúde Coletiva, v.14, n.6, p.2143-2152, 2009. DOI: https://doi.org/10.1590/S1413-81232009000600021

MOYA, J. A.; PARDO, N.; MERCIER, A.. The potential for improvements in energy efficiency and $\mathrm{CO}_{2}$ emissions in the EU27 cement industry and the relationship with the capital budgeting decision criteria. Journal of Cleaner Production, v.19, n.11, p.1207-1215, 2011. DOI: https://doi.org/10.1016/j.jclepro.2011.03.003

PINTO JÚNIOR, A. G. P.; BRAGA, A. M. C. B.. Trabalho e saúde: a atividade da queima de resíduos tóxicos em fornos de cimenteiras de Cantagalo, Rio de Janeiro. Ciência \& Saúde Coletiva, v.14, n.6, p.2005-2014, 2009. DOI: http://dx.doi.org/10.1590/S1413-81232009000600008

PNLA. Portal Nacional do Licenciamento Ambiental. Brasília: Ministério do Meio Ambiente, 2021.

ROCHA, S. D. F.; LINS, V. F. C.; ESPÍRITO SANTO, B. C.. Aspectos do coprocessamento de resíduos em fornos de clínquer: Revisão da Literatura. Engenharia Sanitária e Ambiental, v.16, n.1, p.1-10, 2011. DOI: http://dx.doi.org/10.1590/S1413-41522011000100003

RODRIGUES, M. G.; COSTA, F. J. P.. Economia e opções energéticas. Revista Ibero-Americana de Ciências Ambientais, v.8, n.2, p.289-298, 2017. DOI: http://doi.org/10.6008/SPC2179-6858.2017.002.0023

STAFFORD, F. N.; RAUPP-PEREIRA, F.; LABRINCHA, J. A.; HOTZA, D.. Life cycle assessment of the production of cement: a Brazilian case study. Journal of Cleaner Production, v.137, p.1293-1299, 2016. DOI: https://doi.org/10.1016/j.jclepro.2016.07.050

TRISTÃO, J. A. M.; TRISTÃO, V. T. V.; FREDERICO, E.. O processo de reciclagem do óleo lubrificante. Revista IberoAmericana de Ciências Ambientais, v.8, n.2, p.224-238, 2017. DOI: http://doi.org/10.6008/SPC21796858.2017 .002 .0018

A CBPC - Companhia Brasileira de Produção Científica (CNPJ: 11.221.422/0001-03) detém os direitos materiais desta publicação. Os direitos referem-se à publicação do trabalho em qualquer parte do mundo, incluindo os direitos às renovações, expansões e disseminações da contribuição, bem como outros direitos subsidiários. Todos os trabalhos publicados eletronicamente poderão posteriormente ser publicados em coletâneas impressas sob coordenação da Sustenere Publishing, da Companhia Brasileira de Produção Científica e seus parceiros autorizados. Os (as) autores (as) preservam os direitos autorais, mas não têm permissão para a publicação da contribuição em outro meio, impresso ou digital, em português ou em tradução. 